\title{
Discovery and Format \\ of Input Structures for Tactical Generation
}

Mark Seligman

ATR Interpreting Telecommunications Res. Labs.

2-2 Hikaridai, Seika-cho, Soraku-gun, Kyoto 619-02 Japan

email: seligman@itl.atr.co.jp

\begin{abstract}
We are concerned with the proper form for the structures which serve as the output of strategic generation and the input to tactical generation. How should the elements of this structure be discovered and how they should be arranged? In both respects, our proposals will contrast with those of Rhetorical Structure Theory [Mann and Thompson]. Major components of the structure are discourse relations and "rhetorical types" (pragmatic objects similar to speech acts) to be discovered via paraphrase relations. We describe a discovery procedure and compare it with similar proposals by Ivir et al and Knott and Dale. We then argue that these and other components, once identified, should be arranged in lattices rather than strictly in hierarchies. As illustration, we list the discourse relations and rhetorical types needed to generate a paragraph-length discourse studied by Mann and Thompson. We present a lattice composed of these, and give one example of an alternate version of the discourse which could be generated from it.
\end{abstract}

\section{INTRODUCTION}

Our concern here is with the proper composition and form of the structures which serve as the output of strategic generation and the input to tactical generation -- that is, as the interface between these processes. We take discourse relations (essentially semantic meta-relations) and "Ihetorical types" (pragmatic objects similar to speech acts) to be major elements of tactical input. Our brief is that these should be discovered via judgments of synonymy, and should be arranged in networks or lattices rather than exclusively in hicrarchies.

Section 1 motivates and describes a paraphrase-oriented discovery method based on a suggestion by [Ivir et al 1973] and compares it with a similar proposal [Knott and Dale 1992]. Section 2 discusses rhetorical types and their discovery. Section 3 compares the paraphrase-based point of view with that of theories in the style of RST which concentrate on elucidating the speaker's underlying rhetorical goals. A general theme will be that much information concerning rhetorical goals and strategy should remain beyond the view of tactical generation programs. Section 4 defends the departure from strict hierarchy in our discourse networks. Finally, section 5 proposes a complete lattice as tactical generation input for a paragraph-length discourse studied by [Mann and Thompson 1983, 1984, 1985] and gives one example of an altemate version which could be generated. The discourse relations and thetorical types we feel are needed to generate this discourse are listed in an Appendix I. Each is accompanied by several possible expressive structures.

We will be describing no particular programs or processes. We are concerned with strategic programs only in attempting to influence their final output. Concerning tactical generation procedures suitable for expressing the lattice of Section 5, see [Seligman 1991].

\section{PARAPHRASE-BASED DISCOVERY OF DISCOURSE RELATIONS}

We bclieve that "discourse relations" (essentially, semantic meta-rclations) and "rhetorical types" (ruughly, specch acts) 1 are major elements of an appropriate input structure for tactical generation, and that both sorts of elements can be identified by analyzing their surface linguistic expressions. [Seligman 91] presented a discovery methodology based on synonymy judgments, one intended to refine and make more explicit an earlier proposal appearing in [Ivir et al 1973]. Recently, an independent but quite similar suggestion by [Knott and Dale 1992] has introduced some further worthwhile refinements.

Let us put forth the view of tactical generation which motivates this opinion. We can then go on to compare the three procedures.

We take it as fairly uncontroversial that the role of a tactical generation program is to express certain preselected semantic and pragmatic structures. The strategic component has decided that certain meanings and functions are to be expressed; and the tactical component's assignment is to select any combination of expressors which preserve that meaning and function. Strategy has decided what to say; tactics must choose a way of saying it.

But certain implications of this tactical assignment -"select expressors so as to preserve meaning and function" -need clarification.

One implication, we think, is that a semantic or pragmatic symbol at the tactical level should be considered by its very definition a shorthand for a semantic or pragmatic equivalence relation: a way of capturing what semantic or pragmatic characteristics certain surface expressions have in common. And so a tactical generator's task with respect to such a symbol, stated a bit more carefully, is to choose among expressions which have been previously judged semantically or pragmatically equivalent in context and grouped under that symbol for just that reason. (And, since there will be many symbols, each with several possible structures, it must also make sure that the respective expressors are compatible.)

From this reconceptualization or redefinition of the tactical role, it follows directly that a procedure for discovering semantic or pragmatic symbols at the tactical level should depend on speakers' semantic and pragmatic equivalence judgments.

A commitment to restrict the tactical role to making expressive selections and checking expressor compatabilities has a second implication: while there is a great deal to be known about the connection between tactical input symbols and communicative goals or cognition, it is not the tactical generator's job to know it. Its input symbols should reflect utterance goals or the processes of deciding what to say only to the extent that these are slated for explicit expression -- for instance, as the explicit markings of this or that speech act. A strategic program, when delivering an input structure to the tactical generator, should supply in its symbols and their interrelationships all the information necessary to make meaning-or-functionpreserving expressor choices, but it need not supply a full trace of the strategic reasoning, and we should not expect to 
read the speaker's full motives, word selection process, and so on in the tactical input without reading between the lines. When the fox tells the crow his voice is beautiful, we will see nothing of his ulterior motives in the tactical generation input, though presumably they lurk in prior structures leading to it. At that level, we see only an assertion, indicating some speech goals to be sure, but only such as the fox decided would be politic.

We are not, of course, suggesting that strategic problems be ignored -- only that they can and should be cleanly separated from tactical ones. It is clear that true semantic and pragmatic studies -- studies showing how a given meaning or function (set of equivalent expressors) is selected in context -- must inform strategic programs. (Beyond this, they are desirable for tactical generation efforts as well, since semantic/pragmatic analysis can make more plausible a set of discourse relation symbols discovered via speakers equivalence judgments. For this purpose, we semantically analyze several relations in [Seligman 1991] comparing our analysis with several competing ones.) But while semantic/pragmatic study is indispensable, it is methodologically valuable to separate it from synonymbased discovery of relations and to treat the discovery as primary, as a first step. One can first determine that native speakers judge several expressions to have the same meaning or function in certain contexts, and then use this information as a guide in investigating the nature of the equivalence -- the linkage to situations and goals.

Assuming that the purpose of tactical semantic and pragmatic symbols is precisely to capture paraphrases -- to symbolize what is semantically and pragmatically invariant in a range of surface expressions -- it is natural that a symbol set is to be tested by generating purported paraphrases from it. Various versions of the output should indeed be judged to preserve meaning and pragmatic function. If not, another cycle of elicitation must tease apart distinctions or commonalities missed the last time round. Such iterative semantic investigation is not new, of course: it has been termed experimental semantics by [Hutchins 1971, 1975. Leech 1970, 1974, Mel'chuk and Zholkovski 1970] and others. The present modest proposal is to extend this sort of investigation to discourse relations and rhecorical types as well as to relations in general. And in fact, we will characterize discourse relations as meta-relations, not different in kind from the lower-order relations they dominate.

We now examine and compare three specific paraphrasebased discovery procedures for discourse relations. We can roughly characterize these as semantic meta-relations -second-order relations, relations which relate relations .. postponing further discussion until the discovery procedures have been introduced.

\section{THE S-RELATORS OF IVIR ET AL}

In a study unfortunately little known, [Ivir et al 1973] categorize with uncommon completeness numerous " $S$ relators" -- syntactic structures associated with identifiable semantic links among the meanings of sentences or clauses. Their catalog was compiled as part of a contrastive study of English and Serbo-Croatian, and thus strives for crosslinguistic universality.

They began their study by compiling a list of surface clause relators from the Brown corpus and standard grammatical sources. Their method for discovering discourse relations by comparison of such surface expressions is described only as follows:

... we chose sets of two sentences [in each language] and applied the test of whether a particular item [surface structure] could link them without substantially altering the relationship indicated by other members of the same category. What exactly constituted a "substantial" alternation, of course, presented a complex problem, which we dealt with by appealing to the intuition of native speakers IIvir et al 1973:29].

The resulting S-relators, some 62 of them, are listed with testing frames taken to be diagnostic and sample relating structures. Here are two, omitting the equivalent SerboCroatian:

\section{Centrast}

Relator implies that $S 2$ contrasts strongly with $S 1$.

That material is rather heavy. This material is light.

Inlby (way of) contrast

Manner of causation

Relator implies that S2 is brought about by means of S1.

He lost control of the car. He knocked down the power line.

thus

and so

in this way/manner

thereby

that is how/the way

Other relators, intuitively grouped in the study according to semantic field, include NON-REAL.CAUSE (as if), COMPARATIVE.DEGREE ( so ... that, to such a degree), and fifty-odd others. All these relations are listed along with a few typical expressors in an appendix to [Seligman 91]. Later studies were planned to provide fuller expressor listings, detailed syntactic analysis of the expressors, and stylistic comments concerning them, c.g. "colloquial use only". However, it has not been possible to obtain them or determine if they were completed.

[Seligman 1991], while accepting the basic premise of the procedure just described, attempted to fill it out -- to make it more explicit and complete. In particular, Ivir et al's brief methodological description left unclear how expressive structures can be discovered which are not simple conjunctions. Our procedure is designed to enable such discovery by eliciting free paraphrase in addition to simple syntax-preserving substitutions.

The following methodology was suggested:

- Compile a list of surface clause relators. Make it as complete as possible.

- From native speakers, elicit plausible examples for each expressive structure. For because, the example Fred failed the test because he didn't study might be elicited.

- Elicit paraphrases of the examples, stipulating that the meaning should remain unchanged so far as possible. (We take the position that, while true paraphrase may 
be rare owing to differences in emphasis, associations, register, etc., truth value can indeed remain invariant among a series of alternate utterances. There is some evidence [Leech and Pepicello 1972] that speakers can observe such distictions. See also [Leech 1974] and [Seligman 1979].) For the present example, the following paraphrases might be among those elicited:

Because Fred didn't study, he failed the test.

Fred failed the test because of not studying.

Fred failed the test due to not studying.

Fred did not study, so he failed the test.

Fred did not study. Consequently, he failed the test.

Fred's not studying caused his failure on the test.

Fred's failure on the test was the result of his not studying.

- The paraphrases may bring to light expressive structures other than clause relators, e.g. because of and due to, which relate clauses to noun phrases. Add these to the master list, and repeat the above steps for them.

- Paraphrases based on a given structure (like because) may employ other structures in the master list (like so, consequently, etc.). By cross-correlating these patterns, sort the expressive structures into proposed synonymy groups.

\begin{tabular}{|c|}
\hline 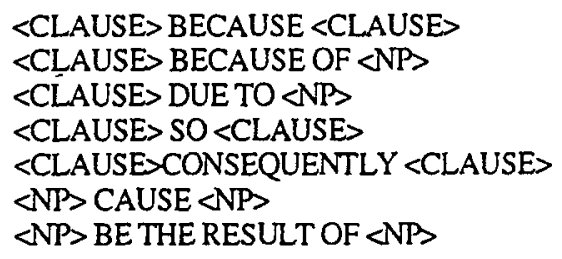 \\
\hline \\
\hline
\end{tabular}

- Assign a name to the relation assumed to underlie each such group -- in the present case, CAUSATION is a good mnemonic candidate, though RELATION.1 would also do -- and the preliminary semantic analysis of discourse relations is complete.

Notice that a given expressive structure can belong to several synonymy groups -- can be polysemous, in other words. The expression so, for example, can express not only CAUSATION (He lost too much blood so he died) but also REASON (I'm studying so I won't flunk).

\section{KNOTT AND DALES PROCEDURE}

[Knott and Dale 1992] independently proposed a discovery procedure for discourse relations which is quite similar to the [Seligman 1991] proposal just discussed.

\begin{abstract}
... a classification of phrases according to their function in discourse is our central objective. ... In keeping with the data-driven methodology adopted thus far, the classification will be made by means of a simple linguistic test, rather than by making theoretical claims about the scmantics or pragmatics of the phrases in the corpus. The test is to do with substitutability. Very broadly, if two phrases are intersubstitutable in a passage of discourse than they should be classified in the same category. [Knott and Dale 1992:18]
\end{abstract}

The study also goes beyond the [Seligman 1991] proposal in some respects. The most important refinement permits the principled discovery of a hierarchical taxonomy of discourse 3 relations, rather than the flat set of categories given by our procedure or the intuitively arranged taxonomy of Ivir et al. The key is to note relations of hyponymy as well as equivalence among the expressions studied.

If one phrase can always be substituted for another, but not vice versa, then the latter phrase should be classified in a category subordinate to that of the former phrase. In this way a taxonomy of synonyms and hyponyms can be constructed. [Knott and Dale 1992:18]

This addition is quite valuable. First, it promises to display relationships among discourse relations more clearly and economically. Representation of these relations in a framebased inheritance hierarchy becomes increasingly attractive, for instance. (The advantages of this representational format for linguistic objects in general are argued throughout [Seligman 1991].) But it also points up once again an underlying similarity between discourse relations and relations in general: discourse relations are relations, and subject to the same analytical techniques. Hyponymy relations, long noted among lexical elements, can be recognized among discourse relators as well.

Recognizing such hyponymy relations among discourse relators, we realize that one strategic task is to choose a degree of specificity for relations at the discourse level as for first order relations. The choice between in short or the more specific in conclusion, then, is similar to that between break and the more specific shatter. At the extreme, certain expressors like full stop or semicolon would seem to be so general as to express only that some unspecified relation obtains.

On the other hand, another feature of the $K \& D$ procedure may detract from its flexibility and generality. Like the Ivir et al procedure described above, the Knott and Dale substitutions preserve syntactic structure. While their procedure does permit appropriate variation in the position of a substitute expression, it does not accommodate (as our procedure does) very free paraphrases in which a relating expression is, for example, sometimes a coordinator, sometimes a verb, sometimes a preposition, etc., with appropriate syntactic changes in the related elements (John is fat because he eats too much. John's overeating causes his obesity. John is fat because of his overeating.) Thus, whereas our procedure starts with conjunctions and spreads toward meaning-related expressions of any class, that of Knott and Dale purposely begins with and keeps to coordinators, subordinators, conjunct adverbs, and certain phrases taking sentential complements. (A clear procedure is described for selecting these relators at the outset [Knott and Dale 1992:14-15].) A compromise procedure which allows free paraphrases while recognizing hyponyms might provide the best of both worlds.

Based upon this methodology for discovery, we believe with Ivir et al and with Knott and Dale that many discourse relations can be recognized. (Ivir et al list more than 60 ; $K \& D$ group relators into about 45 categories.) Recall that we characterized discourse relations as meta-relations. Speakers of a language recognize and conventionalize thousands of first-order relations; why should they not also recognize many relations among relations? Accordingly, in the terms of [Hovy 1990], we are: "profligate" seekers of discourse relations. (By contrast, the relatively "parsimonious" Mann and Thompson posit some 25-30, while Hobbs employs only eight.) We would not be surprised if there were a hundred or more, considering that we recognize additional 
distinctions beyond those seen by Ivir et al. (Specifically, we see numerous fine distinctions within the semantic field of but, however, etc. which Ivir et al do not observe. They list seven relations where we see 15-20 [Seligman 1986].)

We now turn from discourse relations toward a different class of linguistic objects, roughly that of speech acts.

\section{RHETORICAL TYPES}

To realistically generate paraphrases of a sample discourse below, we will need to recognize semantic/pragmatic objects we term rhetorical types. Following are the textual segments in question, each accompanied by paraphrases.

- Tempting as it may be, we shouldn't embrace every popular issue that comes along.

Although, granted, it is tempting, we shouldn't embrace every popular issue that comes along.

I grant you that it is tempting, but we shouldn't embrace every popular issue that comes along.

True, it is tempting, but we shouldn't embrace every popular issue that comes along.

- Let's be clear: I personally favor the initiative and ardently support disarmament negotiations to reduce the risk of war.

Let me make one thing perfectly clear: I personally favor the initiative and ardently support disarmament negotiations to reduce the risk of war.

I personally favor the initiative and ardently support disarmament negotiations to reduce the risk of war. I want that to be quite clear.

- I urge you to vote against a CCC endorsement of the Nuclear Freeze Initiative.

I request that you vote against a CCC endorsement of the Nuclear Freeze Initiative.

I ask you to vote against a CCC endorsement of the Nuclear Freeze Initiative.

We establish paraphrase groupings here in order to distinguish rhetorical types, just as we did for discourse relations -- and just as we might do for semantic objects in general. The more reliably we can predict a paraphrase set (e.g. true, <clause> or granted, <clause>, etc.), the more certain we can be that a distinct underlying pragmatic object has been identified. We will refer to the symbol yielding the first grouping as CONCESSION. The second and third groupings will respectively be generated via the symbols CLARIFICATION and FORMAL. REQUEST.

But just what are rhetorical types? And how are they different from standard relations like BELIEVE or discourse relations like NON.PREVENTIONS? We now sketch our point of view.

For any utterance, we can attempt to specify the circumstances that make it appropriatc. The circumstances, however, divide themselves into two important types: those relating to the communicative goals of the speech participants, which we can term rhetorical circumstances; and those relating to everything else, which we can term non-rhetorical circumstances. (Non-rhetorical circumstances are sometimes called "semantic" in current discussion. We avoid this term here as overburdened.)

Specification of non-rhetorical circumstances alone will not suffice to predict the felicity of any utterance. Having determined that the speaker's aunt's pen is on the table, we cannot predict the appropriateness of her utterance $M y$ aunt's pen is on the table. Rather, the utterance will be appropriate if these non-rhetorical circumstances obtain and the speaker believes the audience does not know about them, ought to know about them, etc. -- in other words, when all the rhetorical circumstances (felicity conditions) for an assertion also obtain [Searle 1969]. In fact, it is just because a great many utterances share these same rhetorical circumstances that we find it worthwhile to distinguish a subclass of utterances called ASSERTIONS. Other utterance subclasses specified in terms of the rhetorical circumstances they share are recognizable as speech acts in the tradition of [Austin 1962] and [Searle 1969] .. assertions, questions, commands, requests, etc. We will instead speak of "rhetorical types", however, because we want to include utterance classes not universally considered in the speech act literature. In the cases at hand, these are subclasses of "classical" speech acts. For example, FORMAL.REQUESTS can (obviously enough) be seen as a subclass of REQUESTS. Similarly, we view CONCESSIONS as a subclass of ASSERTIONS in which the specification is added that the speaker grudgingly recognizes the truth of the conceded assertion. And we view CLARIFICATIONS as a subclass of ASSERTIONS in which the speaker explicitly signals apprehension that the assertion is needed to clear up misunderstandings which may have been engendered by the foregoing.

Do discourse relations represent groupings of rhetorical or non-rhetorical circumstances? One is apt to opt for Rhetorical at first blush: they do help to hold a discourse together by relating relations -- hence the name discourse relations -- and thus acquire an association with pragmatics. But clearly, no communicative goals need be considered to recognize a CAUSATION or a SEQUENCE. In our conception, then, discourse relations categorize nonrhetorical utterance circumstances just as garden-variety relations do, and are not to be confused with rhetorical types.

Bclow, we symbolize the relationship between a thetorical type and an associated relation (of any order) as one of dominance. ASSERTION.1, for instance, might dominate HITTING.1. Other possible representations are considered in [Seligman 1991].

\section{PARAPHRASE-BASED DESCRIPTIONS VS. RST}

Having described an approach to discourse relations and rhetorical types (finely subcategorized speech acts), we are now in a position to very briefly discuss the relationship between these objects and those of certain current discourse theories. We concentrate on the Rhetorical Structure Theory of [Mann and Thompson 1983, 1984, 1985] as especially influential, but our comments will apply equally to related theorics in which the central concem is an account of the speaker's goals. Knott and Dale provide a good starting point: although they describe their own substitution-based discourse relator categories as "pre-theoretical", they have noted some discrepancies between these categories and the relations of RST.

It should be noted that the categories of cue phrases can often be mapped roughly back onto relations in RST's original set. Relations like sequence, contrast, 
circumstance, cause, and result all find natural correlates in the taxonomy. ... At the same time, there are significant differences. These are sometimes to do with granularity: for instance, cue phrases marking a sequence relation do not form a single group, but are themselves taxonomized. Again, the distinction between volitional-result and non-volitional-result finds no correlate in the taxonomy. On the other hand, some RST relations do not appear to have any relational phrases associated with them. Evaluation, for instance, seems to fall into this class. And elaboration, while a prominent relation in many RST analyses, seems to have no single phrase to mark it -a particularly surprising finding. [Knott and Dale 1992:21]

These mismatches come about naturally. RST is primarily concerned with the speaker's goals. When an analysis is complete, one hopes to have inferred an indication of the underlying goals which gave rise to the discourse. Surface expressions are viewed primarily as clues to these, albeit very imperfect clues in that they are often ambiguous or missing. One views speaker goals through surface expressions as through a glass darkly. So an RST analysis gets what help it can from the surface expressions, but depends mainly upon plan inference. Now, given the aim of finding deeper rhctorical goals and given the indirectness of the link between goals and cue phrases, it should not be surprising that when an RST structure is used for generation, it is very likely to underspecify or not specify the particular language to be used. To the extent that RST-style descriptions concentrate on rhetorical goals like evaluation or elaboration which may never be explicitly expressed, they are more appropriate for strategic than for tactical description.

By contrast, a paraphrase-based structure, when it is used for generation, should indeed give a full specification of the available linguistic choices. But in compensation, as already dramatized with fox and crow, it will not display all the planning that went into the decisions about what to express.

A different metaphor may be helpful in conceptualizing the difference between a goal-oriented description of an utterance and one which is oriented toward explicit linguistic expression. When building a house, the contractor expects a blucprint spccifying its structure in sufficient detail for actual construction, though some degrees of freedom usually remain. One ccrtainly has many goals in building the house, including the meeting of many constraints -- financial ones, those of site geography, and so on. But these do not appear in the blueprint. The blueprint, rather, is the detailed work specification resulting from -- the output of, the trace of -the planning to meet those goals. Of course, one can infer much about the goals by examining this work specification, but does not expect them to be included within it. One can guess a lot about a homeowner's financial constraints by looking at a blueprint; but one does not expect to see there the notation, "Build this house for under X dollars", or the owner's bank balance. Conversely, the builder would not get sufficient guidance for actual construction by looking at a high-level description of the owner's housing goals.

It was said that some degrees of freedom usually remain. Blucprints can vary in the degrees of specification they provide and the degrees of freedom they leave. We can for instance imagine a blueprint as made up of several overlays. There is the electrical wiring diagram, the piping, etc., in addition to the framework. If onc overlay were missing, the builder would be underguided or underconstrained. Likewise, an input structure for tactical generation may ideally contain several overlays, and be underconstrained for the lack of one or more. The network of Figure 4 , in particular, does not indicate thematic information or salience. Consequently, it underconstrains the ordering and thematic structures of the output, giving rise to paraphrases which vary freely in these respects. ([Hovy 1993] urges a many-factored view of generation input.)

Notice finally that the choices of surface expression for discourse relations and rhetorical types available to a tactical program will often include ambiguous expressors. So, as we saw, can express CAUSE, PURPOSE, and other relations. So even a relatively full specification from the generation viewpoint can lead to language which would be ambiguous for an analysis program. Expressors can also be unhelpfully general in meaning from an understander's viewpoint, with full stop as an extreme example. An analysis routine may in such cases need a wide range of knowledge to determine which tactical symbol gives a better interpretation, or what more specific symbol could have been used instead. However, knowledge of possible mappings between expressors and semantic/pragmatic symbols, and of the full range of conventional discourse relations and rhetorical types, should provide some constraint. To this extent, knowledge about symbols at the tactical level should give at least some help in ultimately working back toward the rhetorical goals that gave rise to the discourse.

\section{HIERARCHIES OR NETWORKS?}

We have discussed symbols for discourse relations and rhetorical types in tactical input structures. We turn now to the arrangement of these elements. To give a preliminary idea of our motivations for employing graphs rather than hierarchies, consider the story of Ted, who drank too much and got sick, but gamely went to work anyway. Figure 1 (top) shows our preferred representation for the underlying set of relations. A fuller account of the relations CAUSATION and NON.PREVENTIONS appears in [Seligman 1991]. We assume they are sufficiently self-explanatory for the present. For comparison, Figure 1 (bottom) displays a hierarchical version of the same set of relations.

We would argue that the hierarchical version distorts the relationships in question. It was not the causal relation between Ted's drinking and becoming sick that failed to stop him from heroically going to work. It was his becoming sick which failed to prevent his going ahead. However, if we are bound by convention to offer only hierarchical representations for this complex of relations, there will be no way of avoiding such distortion. It is this convention we are arguing against.

We do not argue that discourse segments can never take on a hicrarchical form. The point is rather that hierarchies appear as special cases in relation networks, and that nonhierarchical connections can be formed as well.

Let us reconsider the same points in the context of a somewhat larger and less artificial network. We will consider a fragment from a text studied by Mann and Thompson, given in full in Section 5 .

Tempting as it may be; we shouldn't embrace every popular issue that comes along. When we do so, we use precious limited resources where other players with superior resources are already doing an adequate job. Rather, I think we will be stronger and more effective 
if we stick to those issues of governmental structure and process, broadly defined, that have formed the core of our agenda for years. Open government, campaign finance reform, and fighting the influence of special interests and big money -- these are our kinds of issues.

M\&T's analysis of the fragment appears in Figure 2. For comparison, our own analysis appears in Figure 3.

M\&T see this entire fragment as instantiating a single discourse schema, THESIS/ANTITHESIS. Thus the fragment divides itself into something the writer identifies with and something he does not identify with.

In our analysis, by contrast, there is no overarching THESIS/ANTITHESIS relation. The various relations in the fragment are linked together, to be sure; but it is not because they are all under a single roof. Rather, they are linked as towns are linked in a road system: any destination can be reached from any other, sometimes in more than one way.

Compare also the EVIDENCE relation of M\&T to the REASON.FOR.BELIEF relation of our analysis. In both analyses, there is a claim to be supported, namely the proposition we shouldn't embrace every popular issue that comes along. We see only the SHOULDN'T proposition as the claim. But M\&T treat this proposition as embedded within another relation, so that the latter is represented as the thing to be proved. We find this indirectness unintuitive, an artifact of the dictum that only hierarchical arrangements are allowed. (Similarly, we earlier rejected a straw-man analysis of the Ted discourse in which the relationship between NON.PREVENTION.1 and BECOMING.1 was obscured by an intervening layer of structure.)

With this orientation, we are now prepared to present several arguments against a purely hierarchical discourse representation.

- The existence proof. Some discourses seem coherent because there are interconnections among their major parts, even though there would seem to be no identifiable overarching schema in which they all participate. We offer the Ted mini-discourse, Ted drank too much, so he got sick, but he went to work, as an example. It secms to be organized more like a three-link chain than a trec. If this is granted, we will have found at least one example of a coherent but non-hierarchical discourse.

- The reductio ad absurdum. We doubt that a single overarching schema can be discovered for a very long discourse like a novel. If one can be found, we are afraid it will be too general to be useful. In particular, it is not likely to have expressions predictably associated with it, once upon a time notwithstanding. It might be countered that discourses are hicrarchical only up to a certain size. But as soon as one grants the need for some non-hierarchical mechanism to relate the largest hierarchics, our case is made.

- The handling of reference. Graphs arc often preferred over trees to represent situations in which a single entity performs multiple roles. In the CCC Text, for example, the proposition/relation underlying (we) embrace every popular issue that comes along plays a part in three higher-level relations: it is tempting to ..., we shouldn't ..., and if ... then we will use limited resources. Our representation handles this multi- 6 reference quite naturally, by having three different links converging on the same relation. There can be no converging in the hierarchical representation. Indeces could be used to show the convergence; but this is extra machinery we would rather do without.

- Directness of connections as an aid to record-keeping. M\&T recognize that within any span there is a proposition that epitomizes its function [Mann and Thompson 1984:6]. We think that such a most-functional proposition should itself be recognized as the direct argument, or terminal, of the relation in question. The relationships of span-mates to the object on the business end of a relation should be represented directly, via other specific relations. Such a network of direct and specific connections will facilitate recordkeeping when discourse segments are scrambled during realization, especially when elements belonging to separate $M \& T$ spans intermix.

- Can there be degrees of coherence? Arguing for an exclusively hierarchical treatment, M\&T argue that, if any single relation is removed, the network as a whole is split into pieces: it is no longer coherent [Mann and Thompson 1985:12]. In our treatment, a network may or may not be sundered when a single link is cut, since there may be alternative routes available between subnets. Its coherence will indeed be reduced, since one less relation is made explicit, but this is a matter of degree. And it should be. A discourse, it seems to us, is not usually judged either totally coherent or totally incoherent. Rather, it may be more or less coherent.

A network analysis does not preclude the recognition of hierarchical part-to-whole relationships within a discourse. On the contrary, the structure appears in the net's topology. Consider a transportation network, in which roads cluster and converge to form obvious hubs, linked by sparse trunk lines. In a discourse network, relations likewise define bustling hubs and lonely highways. The hubs, or sets of nodes linked by many connections, are major parts or divisions within the net. Relatively few connections run between them. Further, just as there may be sub-hubs within a large city -- the Port Authority terminal in New York City, for example -- discourse network topology can define parts within discourse sections, so that the familiar sort of partwithin-part hierarchy can be recovered. Further, discourse parts or segments, once identified in this way, might certainly be associated on a strategic level with rhetorical purposes in the manner of [Grosz and Sidner 1986].

\section{EXAMPLES}

For illustration, we now tum to a short attested discourse studied by [Mann and Thompson 1983, 1984, 1985] and to description of the discourse relations and rhetorical types we find necessary for tactical generation of it. A sample lattice composed of these will be shown, and one alternate version of the discourse which could be generated, assuming a suitable grammar and procedure, e.g. that of [Seligman 1991].

\section{THE CCC TEXT}

We have already seen part of the "CCC Text". A letter-writer attempts to convince fellow members of California Common Cause to vote down a proposal to support a Nuclear Freeze.

I don't believe that endorsing the Nuclear Freeze Initiative is the right step for California $C C$. 
Tempting as it may be, we shouldn't embrace every popular issue that comes along. When we do so, we use precious limited resources where other players with superior resources are already doing an adequate job. Rather, I think we will be stronger and more effective if we stick to those issues of governmental structure and process, broadly defined, that have formed the core of our agenda for years. Open government, campaign finance reform, and fighting the influence of special interests and big money -- these are our kinds of issues.

Let's be clear: I personally favor the initiative and ardently support disarmament negotiations to reduce the risk of war. But I don't think endorsing a specific nuclear freeze proposal is appropriate for CCC. We should limit our involvement in defense and weaponry to matters of process, such as exposing the weapons industry's influence of the political process. Therefore, I urge you to vote against a CCC endorsement of the nuclear freeze initiative.

\section{DISCOURSE RELATIONS AND RHETORICAL TYPES}

The discourse relations and rhetorical types we will use to represent this text at the tactical level are listed in Appendix I along with very brief characterizations, relevant passages in the CCC Text, and representative (but incomplete) list of expressive patterns, some of which will appear in sample generation output below. ([Seligman 1991] provides much fuller discussion of these and other relations, commenting on the differences between our analysis and that of several other researchers. Space precludes such treatment here.)

The relations are grouped for convenience, e.g. as relations of the but type, the and type, the if type, etc. We make no claims for the present grouping scheme, but do believe with Knott and Dale that relations can be classified according to meaning overlaps and differences. (Ivir et al provide an intuitive taxonomy close to our present one. It appears as an appendix in [Seligman 1991].)

\section{LATTICE FOR TACTICAL GENERATION INPUT AND SAMPLE OUTPUT}

The discourse relations and rhetorical types in Appendix I can be interconnected to compose the lattice shown in Figure 4.

[Seligman 1991] describes a small set of network traversal strategies which permits repcated application of a sentence grammar to generate paragraphs expressing the whole net. An important part of this procedure is the capacity to generate bottom-up -- to find well-formed continuations for partial structures which are alrcady complete. Certain simple facilities for handling anaphora are also specified. Space limitations will not permit description of these expression procedures here. For concreteness and illustration, however, we provide one possible output version, Appendix II.

Numbers in the network show the ordering in which lattice nodes would be visited to produce the discourse variant of Appendix II. Numerous other variants (some shown in [Seligman 1991]) could be produced by varying the traversal ordering and selection of expressors. Next to each discourse relation (underlined once) or rhetorical type (underlined twice) is a parenthesized indication of the pattern used in our sample variant. For instance, example (a) indicates the choice of the pattern labeled (a) to express the EXAMPLE relation. Just below the relation is the chosen expressor itself, e.g. "For example".

Two types of abbreviation in the sample network should be pointed out.

First, in order to concentrate on discourse relations and rhetorical types, we use ad hoc semantic tokens as terminals. These can in turn receive ad hoc mappings to syntactic structures below the clause level. For instance, the ad hoc semantic relation IT.IS.TEMPTING might be realized only as clause: it is tempting <verb phrase, to infinitives. In the sample output text, such fixed text is shown in plain typeface. However, discourse-level structures generated analytically -- our focus here -- are italicized.

Secondly, every relation in our network ought to be associated with a rhetorical type, since purely non-rhetorical circumstances cannot alone justify any utterance. So, just as CONCESSION, CLARIFICATION, and FORMAL.REQUEST point to their respective relations, all other relations should be covered by thetorical types, here probably ASSERTION in each case. For simplicity and readability, however, we treat assertions as implied, or unmarked, rhetorical types.

\section{CONCLUSIONS}

We have argued that a high-level specification of a speaker's goals in producing a discourse is not an appropriate input for a tactical generator. The latter must assume that some process has already decided what to say for whatever reasons, and requires a specification of what to say in symbols closely tied to potentially usable surface linguistic expressions. These symbols should represent pragmatic or semantic ("rhetorical or non-rhetorical") equivalence classes of expressions, and should thus be discovered by using as primary data speakers' equivalence judgments. We offered a discovery procedure based on paraphrase, intended to be more explicit and flexible than a procedure suggested by Ivir et al. We then compared our proposal with a later, independent suggestion due to Knott and Dale, finding that the K\&D procedure's ability to induce hyponymy as well as equivalence relationships was advantageous, but that its consideration of only certain syntactic classes as discourse relation expressors might be overly restrictive.

We presented a number of arguments for tactical input specifications arranged as lattices rather than strictly as hicrarchies.

For illustration, we listed the discourse relations and rhetorical types needed to generate an attested paragraphlength discourse. We proposed a lattice composed of these as tactical generation input, and gave one example of the sort of discourse version which could be generated, assuming a suitable grammar and set of procedures.

\section{REFERENCES}

Austin, J. 1962. How to Do Things with Words. Cambridge, MA: Harvard University Press, 1962.

Grosz, B. and C. Sidner. 1986. Attention, Intentions, And The Structure Of Discourse. Computational Linguistics, $1986,175-203$.

Hovy, E. 1990. "Parsimonious and Profligate Approaches to the Question of Discourse Structure Relations." In Proceedings of the 5th International Workshop on Language Generation, Pittsburgh, PA, June. 
Hovy, E. 1993. "In Defense Of Syntax: Informational, Intentional, And Rhetorical Structures In Discourse." In Intentionality And Structure In Discourse Relations: Proceedings of A Workshop Sponsored By The Special Interest Group On Generation of The Association For Computational Linguistics, Columbus, $\mathrm{OH}$, June.

Hutchins, W. 1971. The Generation of Syntactic Structures from a Semantic Base. London: North-Holland, 1971.

Hutchins, W. 1975. "Subjects, Themes, and Case Grammar."Lingua, 35(2) (1975) 101-133.

Ivir, V., and D. McMillan, T. Merz. 1973. "S-Relators." Yugoslav Serbo-Croatian-English Contrastive Project, 7 (1973), 22-64.

Knott, A. and R. Dale. 1992. Using Linguistic Phenomena To Motivate A Set of Rhetorical Relations. Technical Report Rp-34. Human Communication Research Centre. University Of Edinburgh. 1992.

Leech, G. 1970. Towards a Semantic Description of English. Bloomington: Indiana University Press, 1970.

Leech, G. 1974. Semantics. Baltimore, MD: Penguin Books, Inc., 1974.

Leech, G. and W. Pepicello. 1972. "Semantic vs. Factual Knowledge: an Experimental Approach." In Papers from the Symposium on Limiting the Domain of Linguistics. Milwaukee: Linguistics Department, University of Wisconsin-Milwaukee.

Mann, W. and S. Thompson. 1983. Relational Propositions in Discourse. Information Sciences Institute (ISI) Research Report ISI/RR-83-115. November, 1983.

Mann, W. and S. Thompson. 1984. Discourse Structure for Text Generation. Information Sciences Institute (ISI) Research Report ISI/RR-84-127. February, 1984.

Mann, W. and S. Thompson. 1985. Discourse Structure for Text Generation. Information Sciences Institute (ISI) Rescarch Report ISI/RR-85-155. April, 1985.

Mel'chuk, I. and A. Zholkovski. 1970. "Toward a Functioning Meaning-Text Model of Language." Linguistics, 57 (1970), 10-47.

Searle, J. 1969. Speech Acts. Cambridge: Cambridge University Press, 1969.

Seligman, M. 1979. The Semantic-based Grammar of WJ. Hutchins and Some Current Issues in Linguistic Theory. Technical Report, Dept. of Linguistics, UC Berkeley.

Seligman, M. 1986b. But: What and How It Means. Technical Report, Dept. of Linguistics, UC Berkeley.

Seligman, M. 1991. Generating Discourses From Networks Using An Inheritance-Based Grammar. Dissertation, Dept. of Linguistics, UC Bcrkeley.

\section{APPENDLX I: \\ DISCOURSE RELATIONS AND RHETORICAL TYPES FOR THE CCC TEXT}

Relations of the But Type

For extensive discussion of the relations in this group, see [Seligman 1986].

\section{Inference.denied}

I personally favor the initiative and ardently support disarmament negotiations to reduce the risk of war. $B$ ut I don't think endorsing a specific nuclear freeze proposal is appropriate for CCC.

"X prompts inferences which are denied by $\mathrm{Y}$."

inference.prompter $>$ inference.denier

a. $<$ clause $>$, but <clause $>$

(anaphoric)

b. <clause>. However,/But <clause>

c. <clause $>$. <clause $>$, though/however.

inference.denier inference.prompter

d. <clause>, although/though <clause>

\section{Not.X.but.Y}

... we shouldn't embrace every popular issue that comes along ... Rather, I think we will be stronger and more effective if we stick to those issues ... that have formed the core of our agenda for years.

... I don't think endorsing a specific nuclear freeze proposal is appropriate for CCC. [Rather,] We should limit our involvement in defense and weaponry to matters of process ...

The writer negates a proposition and, furnishing a correction, asserts a sharply contrasting one.

\section{denial>correction}

(anaphoric)

a. <negative clause>. Rather,/Instead, <clause>

\section{correction $>$ denial}

b. <clause $>$. <negative clause $>$

\section{Non.prevention}

I don't think endorsing a specific nuclear freeze proposal is appropriate for CCC, [even though] ... let me be quite clear, I personally favor the initiative ...

"X does not prevent Y." Good indicators for this relation are the felicity of in spite of, despite, even so, anyway.

\section{non.preventer>non.prevented}

a. <clause>, but/yet <clause> anyway/despite this/in spite of this /even so. 
b. <noun phrase> <verb phrase, transitive, negative, verb.constraints $=$ root $=$ prevent $>$

c. In spite of/despite <noun phrase>, <clause>

d. Even though/Although/Though <clause>, <clause>

(anaphoric)

e. <clause>. However, <clause> anyway/despite this/in spite of this /even so.

f. <clause>. Despite this./In spite of this,/Even so, <clause>

non.prevented $>$ non.preventer

g. <clause>, even though/although/though <clause>

h. <clause>, in spite of/despite <noun phrase>

\section{Relations of the If Type}

\section{Future.if}

... we will be stronger and more effective if we stick to those issues ... that have formed the core of our agenda for years.

"If $\mathrm{X}$ happens, then $\mathrm{Y}$ will happen."

\section{condition>conclusion}

a. If <clause>, \{then\} <clause>

(anaphoric) -

b. <clause, modality=possible>. In that case, /ff so, <clause> conclusion>condition

c. $\langle$ clause $>$, if $\langle$ clause $>$

Whenever

When (ever] we do so, we use precious, limited rcsources...

"Every time X happens, Y happens."

reference.event $>$ main.event

a. Whenever/When/Every time/Each time <clause>, <clause>

\section{main.event>reference.cvent}

b. <clause > whenever/when/every time/each time <clause>

\section{Relations of the For Example Type}

\section{Example}

... if we stick to those issues ... that have formed the core of our agenda for years. [For example], Open government, campaign finance reform, and fighting the influence of special interests and big money -. these are our kinds of issues.

One segment supplies a special case of a more general proposition stated by the other segment.

general $>$ example

(anaphoric)

a. <clause>. For example,/For instance, sclause> b. <clause>. <clause>, for example/for instance

\section{example>general}

(anaphoric)

c. <clause>. In general,/Generally speaking,/Generally, $<$ clause>

\section{Term.example}

... matters of process, such as exposing the weapons industry's influence ...

The EXAMPLE discourse relation discussed above links entire relations: I like dogs. For instance, I like collies, cocker spaniels, and poodles. But an exemplifying relation can also obtain between a term representing a class and a collection of terms denoting subclasses or class members, as in I like dogs, such as collies, cocker spaniels, and poodles.

\section{general >example}

a. <noun phrase>, such as/for example/for instance <noun phrase>

\section{Relations of the And Type}

\section{Affirmativeaddition}

(... we shouldn't embrace every popular issue that comes along, [because]) when we do so, we use precious, limited resources ... [and] ... I don't think endorsing a specific nuclear freeze is appropriate for CCC.

Two segments supply closely related information. In The children were shouting and the dogs were barking, for instance, both segments are specifications, on an equal footing, of a general statement like It sure was noisy!

\section{conjoint>conioint}

a. $\langle$ clause $>$, and $<$ clause $>$

(anaphoric)

b. <clause>. Also,/Additionally, /In addition, <clause>

c. $\langle$ clause $>$. $\langle$ clause $>$, too/as well/also

d. <clause>. And <clause>

\section{Relations of the Cause Tupe}

\section{Reason.for.belief}

... we shouldn't embrace every popular issue that comes along. When we do so we use precious, limited resources ...

Onc segment supports (supplies reasons for believing) the claim made by the other.

\section{claim>support}

a. <clause $>$, because/for $/$ since/as <clause>

(anaphoric)

b. <clause>. Because/For/I think so because <clause> 
support>claim

c. <clause $>$, \{and $\}$ so <clause $>$

(anaphoric)

d. <clause>. So/Thus/Therefore/That's why I think (that <clause>

\section{Reason.for.illocutionary.act}

I don't believe that endorsing the Nuclear Freeze Initiative is the right step for California CC. ... Therefore, I urge you to vote against a CCC endorsement of the Nuclear Freeze Initiative.

"X. I say this/ask this/order this/request this because Y." Writer/speaker makes an assertion, asks a question, issues an order, makes a request, etc. An accompanying segment furnishes her motivation for doing so.

\section{illocutionary.act>reason}

a. <clause $>$, because/for $/$ since/as <clause $>$

(anaphora)

b. <clause>. Because/For <clause>

reason>illocutionarv.act

c. $<$ clause $>$, \{and $\}$ so $<$ clause $>$

(anaphoric)

d. <clause>. So <clause>

e. <clause>. Accordingly, <clause>

\section{Rhetorical types}

\section{Clarification}

Let's be clear: I personally favor the initiative ...

The writer signals that the accompanying assertion is a clarification.

clarification $>$ clarified

a. Let's be clear:/Let us be clear:/Let me be clear:/Let me make one thing \{perfectly/quite\} clear:/I want to be \{perfectly/quite\} clear: <clause>

clarified $>$ clarification

(anaphoric)

b. <clause>. I want to make this (perfectly/quite\} clear

\section{Concession}

Tempting as it may be, we shouldn't embrace every popular issuc that comes along ...

The writer signals recognition that the embedded assertion must be admitted.

a. Granted,/True,/No doubt about it,/1 grant you,/It must be granted,/To be sure, <clause>

b. <clause>, I grant you/I grant you that/it must be granted

\section{Formal.request}

Therefore, I urge you to vote against a CCC endorsement of the nuclear freeze initiative.

The speaker formally asks the audience to take the action indicated. The requests which we describe here as formal contain performatives: the requester describes the request in the act of making it (I urge you to ..../I ask you to ...). Conventionalized performatives would seem to be a special class of rhetorical types, while nonce performatives (I'm requesting you to ...) appear to employ only standard semantic relations.

a. I urge you/I ask you <verb phrase, form=to.infinitive>

b. I ask that you <verb phrase, form=plain.infinitive>

\section{APPENDIX II : ALTERNATE VERSION OF THE CCC TEXT}

I don't believe that endorsing the nuclear freeze initiative is the right step for California CC because we shouldn't embrace every popular cause that comes-along. We shouldn't do so although, granted, it is tempting to do so. For whenever we do so, we use precious limited resources where other players with superior resources are already doing an adequate job. Rather, I think we will be stronger and more effective if we stick to those issues of governmental structure and process, broadly defined, that have formed the core of our agenda for years. For example, open government, campaign finance reform, and fighting the influence of special interests and big money ... are our kinds of issues. And I don't think endorsing a specific nuclear freeze proposal is appropriate for CCC, even though, let me be quite clear, I personally favor the initiative and ardently support disarmament negotiations to reduce the risk of war. Rather, we should limit out involvement in defense and weaponry to matters of process, for instance exposing the weapons industry's influence of the political process. So I urge you to vote against a CCC endorsement of the Nuclear Freeze initiative. 


\section{FIGURES}
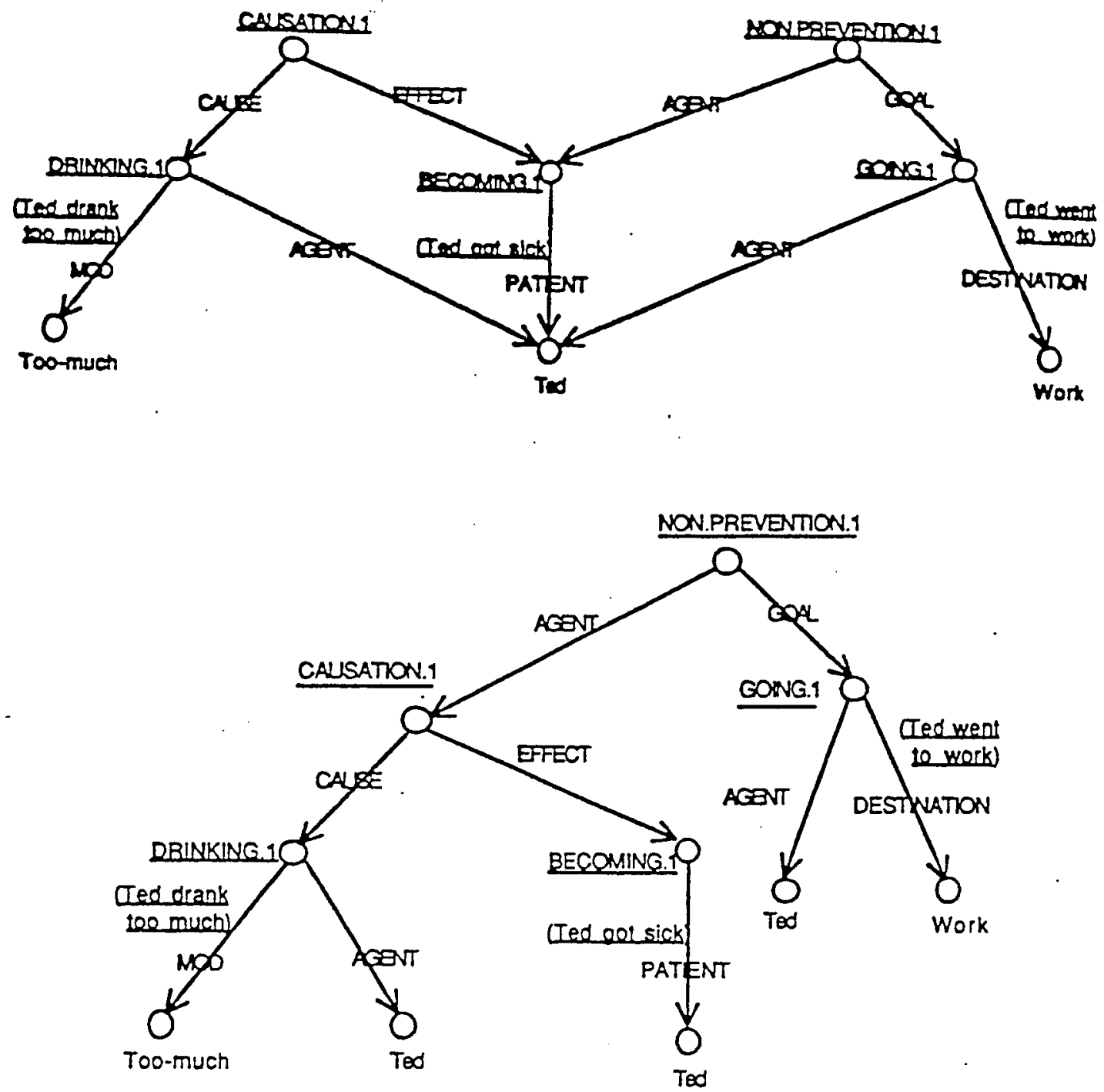

Figure 1: The Ted Discourse, graph style (top) and hierarchical style (bottom)

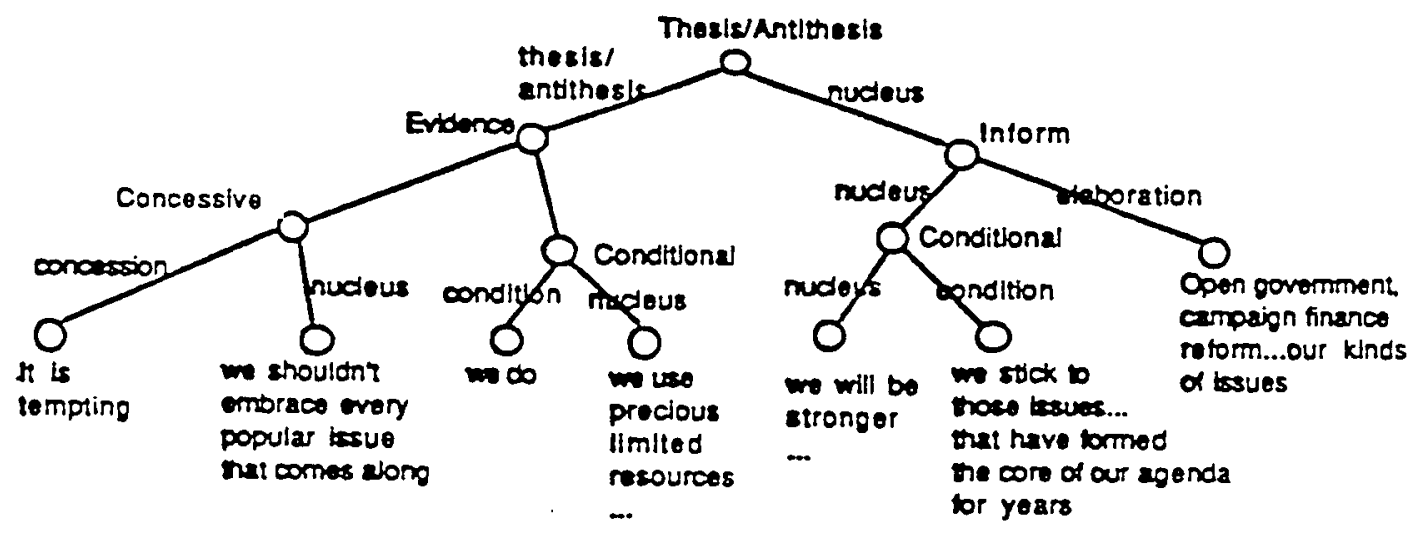

Figure 2: M\&T's analysis of part of the CCC text. 

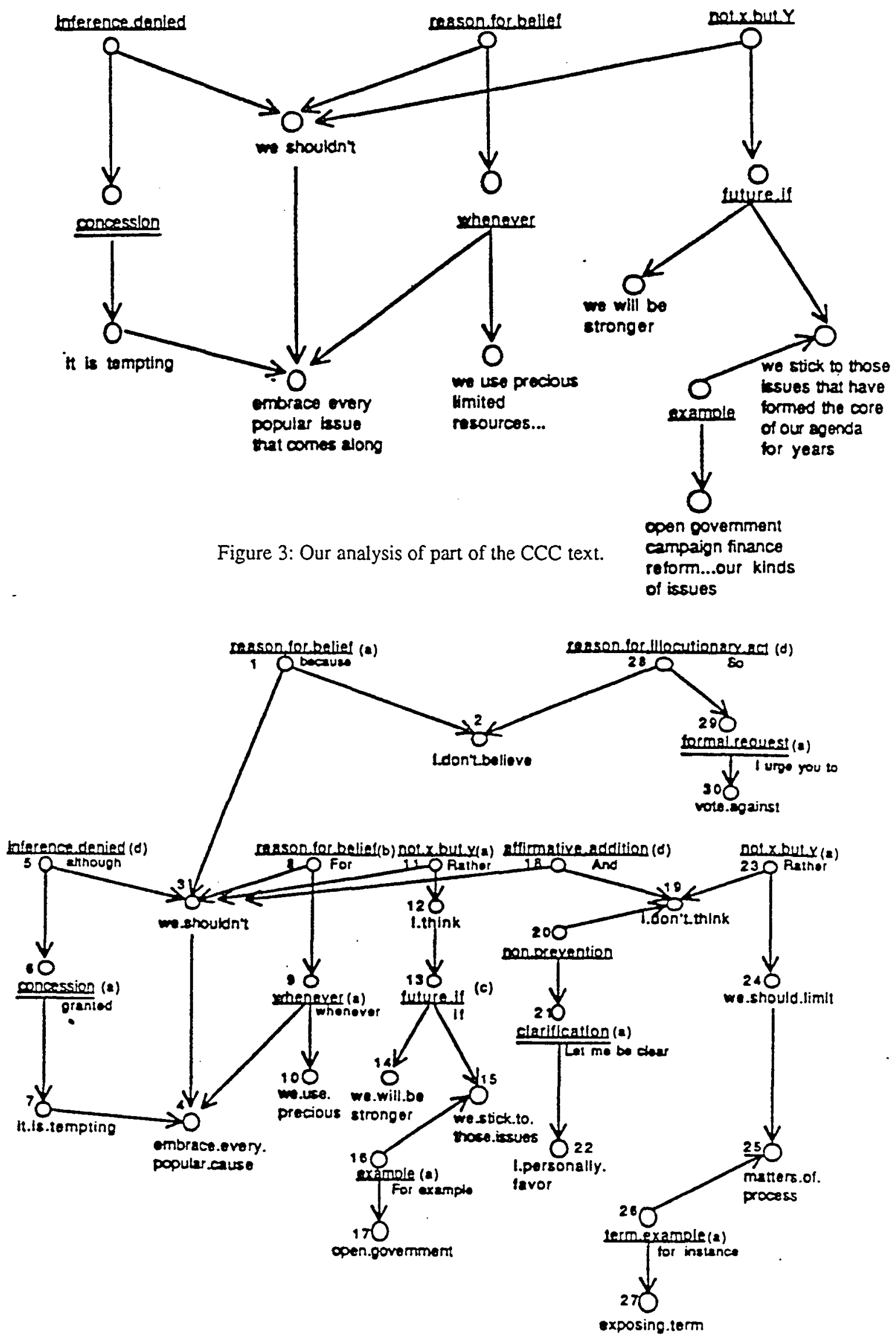

Figure 4: A lattice for the CCC Text, with one possible traversal path marked. 